\title{
Study of Cerebral Venous Trombosis in Central India
}

\author{
Authors \\ Dr Vijay Kumar Nandmer, Dr Ajay Kumar Nandmer
}

\begin{abstract}
Thrombosis of the venous channels in the brain accounts for $0.5 \%$ of all stroke and is an uncommon cause of cerebral infarction relative to arterial disease but is an important consideration because of its potential morbidity ${ }^{l}$ The aim of this study is to evaluate the D-Dimer as a screening tool for the diagnosis of cerebral venous thrombosis.

Aims and Objectives: To study the clinical characteristics, risk factors and outcome of cerebral venous thrombosis. To evaluate the role of D-Dimer in the diagnosis of cerebral venous thrombosis. To find corelation if any between levels of D-Dimer, duration of symptoms and outcome of cerebral venous thrombosis.

Discussion Epidemiology of Cerebral Venous Thromsosis

Age and Sex: In the present study the average age of presentation for patients with cerebral venous thrombosis was 29.79+SD 12.1414 years. The youngest patient was of 14 years while the oldest was of 63 years.

Clinical Features: In the present study headache was the most common symptom seen in $91.6 \%$ of all patients. Other symptoms include seizures (79\%), focal nuerological deficit (50\%), diffuse encephalopathy (29.1\%), fever (29.1\%), and dimunition of vision (8.3\%).

Outcome: In the 24 patients evaluated in our study, 17 (70.83\%) patients had complete recovery in 1 month whereas 5 (20.83\%) had persistent deficit at the end of 1 month. Complete recovery at one month was seen in $80 \%$ of male patients and $78.95 \%$ in female patients. The mortality rate in our study of CVT was found to be $8.33 \%$. Factors associated with increased mortality were presence of sepsis and seizures.

D-Dimer levels in cerebral venous thrombosis: In the present study the D-Dimer levels were found to be elevated in 22 out of the 24 patients evaluated. The mean value of D-Dimer for patients with cerebral venous thrombosis was found to be $1.815417+S D 1.498352 \mathrm{mcg} / \mathrm{ml}$ and that of controls was found to be $0.41433+$ SD $0.234403 \mathrm{mcg} / \mathrm{ml}$. The difference between the values of cases and controls was found to be statistically significant with ' $p$ ' value $<0.001$. Mean (cases)- Mean (controls) $=1.401054$ with $95 \%$ confidence interval between 0.84 to 1.95708 .

Summary \& Conclusions: The present study evaluated 24 patients of Cerebral Venous Thrombosis and 30 age and sex matched controls. The risk factors, clinical presentations, and duration of symptoms of both the groups were determined. The level of D-dimer were determined for both cases and controls and its significance was tested by statistical analysis.

The mean age for cases of CVT in the present study was 29.79 years.

Females accounted for $79.16 \%$ of cases and were affected at a younger age in comparision with males. The male: female ratio in the study was 1:3.8.

Average duration of symptoms before presentation in the present study was $5.67+S D 4.26$ days.
\end{abstract}


Headache and seizures were the most common clinical features of CVT.

In the present study, puerperium was the most common risk factor for the development of CVT.

$D$-Dimer levels were significantly elevated in patients of CVT as compared with controls $(p<0.001)$.

$D$-Dimer assay was found to have high sensitivity (91.66 \%) in predicting the presence of CVT in the present study. The specificity, positive predictive value and negative predictive value of D-Dimer levels was found to be $80 \%, 78.59 \%$ and $92.30 \%$ respectively.

In the present study the sensitivity of D-dimer assay was highest when the test was applied early in the course of illness (100\%). The sensitivity of the test declined rapidly as the duration of symptoms prior to testing increased.

The present study supports the use of D-dimer as a 'rule out' test for CVT. The diagnosis of CVT is unlikely in the presence of a normal D-Dimer value. Patients with features of CVT with elevated D-Dimer should be urgently sent for MRV for confirmation of diagnosis. However the elevation of D-Dimer itself does not indicate presence of CVT.

Keywords -cerebral venous thrombosis, D-dimer.

\section{INTRODUCTION}

In the Indian context, CVT constitutes $10-15 \%$ of stroke in the young and was the commonest cause of stroke in pre-menopausal women.,

Cerebral venous thrombosis (CVT) is associated with a wide spectrum of clinical manifestations, ranging from isolated headache (with or without papilledema) to diffuse or focal brain edema, infarction, or hemorrhage ${ }^{5}$.

Efforts to develop a simple screening laboratory test to detect CVT in patients who present with isolated headache are also a priority.

D-dimer is a fibrin degradation product, a small protein fragment present in the blood after a blood clot is degraded by fibrinolysis. It is so named because it contains two cross linked D fragments of the fibrinogen protein ${ }^{9}$.Thrombosis anywhere in the body activates the coagulation pathway and also the fibrinolytic pathway leading to plasmin mediated breaking of the fibrin clot leading to elevation of fibrin degradation product.

D-Dimer estimation along with application of clinical decision rules is now a standard practice adopted for the diagnosis of deep vein thrombosis and pulmonary embolism. ${ }^{10-12}$ D-Dimer is also used for the diagnosis of other fibrinolytic states like DIC. ${ }^{13}$

The aim of this study is to evaluate the D-Dimer as a screening tool for the diagnosis of cerebral venous thrombosis.

\section{AIMS AND OBJECTIVES}

1. To study the clinical characteristics, risk factors and outcome of cerebral venous thrombosis.

2. To evaluate the role of D-Dimer in the diagnosis of cerebral venous thrombosis.

3. To find co-relation if any between levels of D-Dimer, duration of symptoms and outcome of cerebral venous thrombosis.

\section{MATERIAL AND METHOD}

The study was conducted in the Department of Medicine at Gandhi Medical College \& Hamidia Hospital, Bhopal .The study subjects were selected from amongst the patients who attend the Medicine OPD, Special Clinics or Medical Emergency Ward or were admitted in the medical wards in Hamidia Hospital, Bhopal during the period from October 2010 to October 2011.

Type of Study: Observational Cross-Sectional Study and Case Control Study

\section{Inclusion Criteria}

All patients with confirmed new diagnosis of cerebral venous thrombosis on Magnetic Resonance Imaging or Magnetic Resonance Venogram.

\section{Exclusion Criteria}

- Patients on Anti-coagulation therapy prior to symptoms.

- Patients with coexistent Deep Venous Thrombosis or Pulmonary Embolism. 
- Recent Surgery

- Recent Trauma,

- Liver disease

- Eclampsia

- Disseminated Intravascular Coagulation

\section{Study Design}

The subjects will be selected after applying the aforementioned inclusion and exclusion criteria. A detailed clinical history and complete physical examination will be carried out for each subject in the study as per the predesigned proforma. Age and sex matched controls would be taken all patients. All blood samples would be taken at the first day of diagnosis prior to the initiation of therapy. D-dimer levels were determined for both cases and controls. For determining the association between D-dimer and duration of illness, patients were divided into 2 groups: those with early presentation ( $<7$ days) and those with late presentation and their mean D-dimer levels were determined. For determining co-relation between D-dimer and outcome of CVT patients were grouped as those with complete recovery and those with persistent deficitt 1 month/death. Mean D-dimer levels were determined for both the groups.

\section{Baseline investigations}

- Complete hemogram with ESR and Platelet Count

- Urine examination

- Liver function test with all six components

- Renal function test

- Prothrombin Time and Activated Partial Prothrombin Time

- RA factor and ANA

\section{Imaging Studies}

Magnetic Resonance Imaging/ Magnetic Resonance Venogram :

Confirmation of CVT by either MR imaging with MR venography will be required to consider CVT the established diagnosis.

\section{Measurement of D-Dimer Levels}

D-Dimer would be measured by an automated quantitative latex particle immunoassay. Cut-off values of $0.5 \mathrm{mcg} / \mathrm{ml}$ fibrinogen equivalent units (FEU/ $\mathrm{mL}$ ) for the assay will be used to distinguish positive/negative D-Dimer results, as established in the literature for patients with peripheral venous thromboembolism.

\section{STATISTICAL ANALYSIS}

The data for all the groups will be expressed as Mean+ SD. The sensitivity, specificity, positive predictive value and negative predictive value for D-dimer level would be calculated. The significance of association between D-Dimer levels and Cerebral Venous Thrombosis would be determined by the application of Chi Square Test. The significance of association between d-dimer and duration of symptoms was also determined by students ' $t$ ' test which was used to test co-relation between D-dimer and outcome of CVT .

\section{OBSERVATIONS}

TABLE: 1 Age Wise Distribution of Patients

\begin{tabular}{|l|l|l|l|l|}
\hline \multirow{2}{*}{ Age } & \multicolumn{2}{|l|}{ Cases $(\mathrm{n}=24)$} & \multicolumn{2}{l|}{ Control $(\mathrm{n}=30)$} \\
\cline { 2 - 5 } & No. & $\%$ & No. & $\%$ \\
\hline 0-10 years & 0 & 0 & 0 & 0 \\
\hline 11-20 years & 4 & 16.6 & 5 & 16.6 \\
\hline 21-30 years & 11 & 45.83 & 15 & 50.0 \\
\hline 31-40 years & 4 & 16.6 & 5 & 16.6 \\
\hline 41-50 years & 2 & 8.3 & 2 & 6.6 \\
\hline 51-60 years & 2 & 8.3 & 2 & 6.6 \\
\hline 61-70 years & 1 & 4.1 & 1 & 3.3 \\
\hline$>70$ years & 0 & 0 & 0 & 0 \\
\hline Total & 24 & 100 & 30 & 100 \\
\hline
\end{tabular}

Average Age of Presentation $=29.7916+12.1414$ years.

TABLE: 2 Sex Wise Distributions of Cases and Controls

\begin{tabular}{|l|l|l|l|l|}
\hline \multirow{2}{*}{ Sex } & \multicolumn{2}{|l|}{ Cases } & \multicolumn{2}{l|}{ Control } \\
\cline { 2 - 5 } & No. & $\%$ & No. & $\%$ \\
\hline Males & 5 & 20.83 & 5 & 16.66 \\
\hline Females & 19 & 79.16 & 25 & 83.33 \\
\hline Total & 24 & 100 & 30 & 100 \\
\hline
\end{tabular}

TABLE: 3 Presenting Features

\begin{tabular}{|l|c|c|}
\hline \multirow{2}{*}{ Presenting Features } & \multicolumn{2}{|c|}{ Cases } \\
\cline { 2 - 3 } & No. & $\%$ \\
\hline Headache & 22 & 91.6 \\
\hline Seizure & 19 & 79 \\
\hline Focal Deficit & 12 & 50 \\
\hline Diffuse Encephalopathy & 7 & 29.16 \\
\hline Fever & 7 & 29.16 \\
\hline Diminution of Vision & 2 & 8.3 \\
\hline
\end{tabular}


TABLE: 4 Risk Factors for CVT

\begin{tabular}{|l|c|c|c|c|}
\hline \multirow{2}{*}{ Risk Factors } & \multicolumn{2}{|c|}{$\begin{array}{c}\text { Cases } \\
(\mathrm{n}=24)\end{array}$} & \multicolumn{2}{c|}{$\begin{array}{c}\text { Control } \\
(\mathrm{n}=30)\end{array}$} \\
\cline { 2 - 5 } & No. & $\%$ & No. & $\%$ \\
\hline Puerperium & 14 & 58.33 & 13 & 43.33 \\
\hline Dehydration & 12 & 50 & 12 & 40.0 \\
\hline Sepsis & 9 & 37.5 & 7 & 23.33 \\
\hline OCP & 3 & 12.5 & 4 & 13.3 \\
\hline Smoking & 3 & 2.5 & 4 & 13.66 \\
\hline Autoimmune Disease & 2 & 8.3 & 1 & 3.33 \\
\hline Cancer & 2 & 8.3 & 2 & 6.6 \\
\hline IV Drug Abuse & 1 & 4.16 & 0 & 0 \\
\hline None & 1 & 4.10 & 3 & 9.99 \\
\hline
\end{tabular}

TABLE: 5 D-Dimer Levels in Cases and Control

\begin{tabular}{|l|c|c|c|}
\hline D-Dimer Levels & Cases & Control & Total \\
\hline $\begin{array}{l}\text { Positive } \\
(>0.5 \mathrm{mcg} / \mathrm{ml})\end{array}$ & 22 & 06 & 28 \\
\hline $\begin{array}{l}\text { Negative } \\
(<0.5 \mathrm{mcg} / \mathrm{ml})\end{array}$ & 02 & 24 & 26 \\
\hline Total & 24 & 30 & 54 \\
\hline
\end{tabular}

True Positive

Sensitivity of D-Dimer for CVT = x 100

Disease Positive

$=\quad 22 / 24 \times 100$

$=\quad 91.66 \%$

True Negative

Specificity of D-Dimer for CVT 100

Disease Negative

$=\quad 24 / 30 \times 100$

$=80.00 \%$

True Positive

Positive Predictive Value of D-Dimer for CVT =----- x 100

Test Positive

$=\quad 22 / 28 \times 100$

$=\quad 78.57 \%$

True Negative

Negative Predictive of D-Dimer for CVT =----------- x 100

Test Negative

$=\quad 24 / 26 \times 100$

$=\quad 92.30 \%$

Applying the Chi-square test for the above contigency table, the difference in the value of ddimer in cases and controls is statistically significant. $(\mathrm{p}<0.001)$

\section{DISCUSSION}

EPIDEMIOLOGY OF CEREBRAL VENOUS THROMSOSIS

Age and Sex:
In the present study the average age of presentation for patients with cerebral venous thrombosis was $29.79+$ SD 12.1414 years. The youngest patient was of 14 years while the oldest was of 63 years. The average age for presentation in male patients was found to be $40.41+$ SD 16.03 years whereas the average age of presentation for female patients was found to be $26.94+$ SD 8.03 years.

Cerebral venous thrombosis was found to be more common in females as compared with males. Females accounted for $79.16 \%$ of all patients. The male: female ratio in the study was found to be 1:3.8. Hence females are affected more commonly and at a younger age as compared with males.

\section{Risk factors}

The most common risk factor for cerebral venous thrombosis in our study was found to be puerperium $(58.33 \%)$. Other risk factors include dehydration $(50 \%)$, sepsis $(37.5 \%)$, oral contraceptive pills $(12.5 \%)$, autoimmune diseases $(8.3 \%)$, cancer $(8.3 \%)$, intravenous drug abuse $(4.16 \%)$ and none in one patient. (4.16\%)

Nagpal et al ${ }^{139}$ reported puerperium and synthetic steroid contraceptives as the most common risk factor which is similar to the observations made in our study with puerperium accounting for $58.33 \%$ and oral contraceptives for $12.5 \%$ of our patients.

\section{CLINICAL FEATURES}

In the present study headache was the most common symptom seen in $91.6 \%$ of all patients. Other symptoms include seizures $(79 \%)$, focal nuerological deficit (50\%), diffuse encephalopathy $(29.1 \%)$, fever $(29.1 \%)$, and dimunition of vision $(8.3 \%)$.

Fischer et al ${ }^{127}$ in their study evaluated 17 patients of CVT. Presenting complaints included headache $(70 \%)$, focal neurologic complaints (numbness, weakness, aphasia) (29\%), seizure $(24 \%)$, and head injury (12\%). Ninety-four percent of patients had a focal neurologic finding during illness as compared to $50 \%$ in the present study. 


\section{Outcome}

In the 24 patients evaluated in our study, 17 (70.83\%) patients had complete recovery in 1 month whereas $5(20.83 \%)$ had persistent deficit at the end of 1 month. Complete recovery at one month was seen in $80 \%$ of male patients and $78.95 \%$ in female patients. The mortality rate in our study of CVT was found to be $8.33 \%$. Factors associated with increased mortality were presence of sepsis and seizures.

Fischer et al ${ }^{127}$ in their study evaluated 17 patients of CVT reported a mortality rate of $11.75 \%$ which was slightly in excess of the mortality rate of $8.33 \%$ in the present study. Of the patients who survived, $80 \%$ had a good functional outcome as compared to $70.83 \%$ in our study.

Vembu et al ${ }^{121}$ included 71 patients in their study and reported that $50 \%$ of patients recovered within 2-4 weeks, 15 patients $(21 \%)$ recovered within 412 weeks. The total percentage of patients who recovered in their study was $71 \%$ as compared to $70.83 \%$ in the present study.

\section{D-Dimer levels in cerebral venous thrombosis:}

In the present study the D-Dimer levels were found to be elevated in 22 out of the 24 patients evaluated. The mean value of D-Dimer for patients with cerebral venous thrombosis was found to be $1.815417 \pm$ SD $1.498352 \mathrm{mcg} / \mathrm{ml}$ and that of controls was found to be $0.41433 \pm \mathrm{SD}$ $0.234403 \mathrm{mcg} / \mathrm{ml}$. The difference between the values of cases and controls was found to be statistically significant with 'p' value $<0.001$. Mean (cases)- Mean (controls) $=1.401054$ with $95 \%$ confidence interval between 0.84 to 1.95708 . The sensitivity of D-Dimer assay for cerebral venous thrombosis was found to be $91.6 \%$. The high sensitivity of the test makes it a good screening test for patients presenting with clinical features of cerebral venous thrombosis.

The specificity, positive predictive value and negative predictive value of D-Dimer test for the diagnosis of cerebral venous thrombosis was found to be $80.00 \%, 78.57 \%$ and $92.30 \%$ respectively.

\section{D-Dimer level and Duration of symptoms:}

The D-dimer test for cerebral venous thrombosis had the highest sensitivity when patients were tested within 7 days of symptoms onset. All of the 13 patients who presented within the first 7 days of symptom onset had an elevated level of DDimer greater than $0.5 \mathrm{mcg} / \mathrm{ml}$.

The sensitivity of D-Dimer assay within 7 days of symptom onset was found to be $100 \%$ in our study. Of 11 patients with presentation after 7 days of onset of symptoms, 7 had elevated DDimer levels while 4 patients had normal. The sensitivity of the test after 7 days was found to $63.63 \%$. Hence the sensitivity of the test falls rapidly with delayed presentation.

The mean value of D-Dimer in 13 patients presenting within 7 days on symptom onset was found to be $2.738462 \pm$ SD $1.47259 \mathrm{mcg} / \mathrm{ml}$. The mean value of D-Dimer in 11 patients presenting after 7 days on symptom onset was found to be $0.72 \pm$ SD $0.364916 \mathrm{mcg} / \mathrm{ml}$. The difference between the two values was found to be statistically significant with 'p' value $=0.0311$.

Levels of D-dimer and Prognosis of Cerebral Venous Thrombosis:

In the 24 patients evaluated in our study, 17 (70.83\%)patients had complete recovery in 1 month whereas $5(20.83 \%)$ had persistent deficit at the end of 1 month. Complete recovery at one month was seen in $80 \%$ of male patients and $78.95 \%$ in female patients. The mortality rate in our study of CVT was found to be $8.33 \%$. Factors associated with increased mortality were presence of sepsis and seizures.

The mean value of D-dimer in patients with complete recovery was found to be $1.95 \mathrm{mcg} / \mathrm{ml}$ while that of patient with persistent deficit/death was found to be $1.47 \mathrm{mcg} / \mathrm{ml}$. The difference between the D-Dimer value for patients who recovered and those with persistent deficit / death was not found to be statistically significant ( $\mathrm{P}=$ 0.4739).

Pfefferkorn et $\mathrm{al}^{126}$ in their study of 32 patients reported that the absolute levels of d-dimer did not have any significant correlation with severity of disease or symptoms. 
Misra et $\mathrm{al}^{116}$ reported that D-dimer positivity did not correlate with the extent of venous sinus thrombosis $(\mathrm{P}=0.93)$ and with 3 month outcome as assessed by the Barthel index score $(\mathrm{P}=0.38)$ which corroborated with the findings of the present study.

\section{SUMMARY \& CONCLUSIONS}

The present study evaluated 24 patients of Cerebral Venous Thrombosis and 30 age and sex matched controls. The risk factors, clinical presentations, and duration of symptoms of both the groups were determined. The level of D-dimer were determined for both cases and controls and its significance was tested by statistical analysis.

The mean age for cases of CVT in the present study was 29.79 years.

Females accounted for $79.16 \%$ of cases and were affected at a younger age in comparision with males. The male: female ratio in the study was $1: 3.8$.

Average duration of symptoms before presentation in the present study was $5.67+\mathrm{SD}$ 4.26 days.

Headache and seizures were the most common clinical features of CVT.

In the present study, puerperium was the most common risk factor for the development of CVT.

D-Dimer levels were significantly elevated in patients of CVT as compared with controls $(\mathrm{p}<$ 0.001).

D-Dimer assay was found to have high sensitivity $(91.66 \%)$ in predicting the presence of CVT in the present study. The specificity, positive predictive value and negative predictive value of D-Dimer levels was found to be $80 \%, 78.59 \%$ and $92.30 \%$ respectively.

In the present study the sensitivity of D-dimer assay was highest when the test was applied early in the course of illness $(100 \%)$. The sensitivity of the test declined rapidly as the duration of symptoms prior to testing increased.

D-Dimer levels had no significant co-relation with outcome of cerebral venous thrombosis in the present study $(\mathrm{p}=0.47)$
The present study supports the use of D-dimer as a 'rule out' test for CVT. The diagnosis of CVT is unlikely in the presence of a normal D-Dimer value. Patients with features of CVT with elevated D-Dimer should be urgently sent for MRV for confirmation of diagnosis. However the elevation of D-Dimer itself does not indicate presence of CVT.

\section{BIBLIOGRAPHY}

1. Marie-Germaine Bousser, José M Ferro. Cerebral Venous Thrombosis: An Update. Lancet Neurol 2007; 6: 162-70

2. Ribes MF. Des recherches faites sur la phlébite. Revue Médicale Française et Etrangère et Journal de Clinique de l'Hôtel-Dieu et de la Charité de Paris 1825; 3: 5-41.

3. Nagaraja D, Tally AB. Stroke in the young in progress in Clinical Neurosciences Edt publ Sinha KK, Ranchi 1988; 2:129-45

4. Chopra JS, Prabhakar S. Clinical features and risk factors in stroke in young. Acta Neurologica Scandivia 1979; 43: 289-300

5. Ameri A, Bousser MG. Cerebral venous thrombosis. Neurol Clin 1992; 10: 87-111

6. Einhaupl KM, Villringer A, Meister W, Mehraein S, Garner C, Pellkofer M, Haberl RL, Pfister HW, Schmiedek P. Heparin treatment in sinus venous thrombosis. Lancet 1991; 338 (8767): 597-600

7. Ferro JM, Canhao P, Stam J, Bousser MG, Barinagarrementeria F. Prognosis of cerebral vein and dural sinus thrombosis: results ofthe International Study on Cerebral Vein and Dural Sinus Thrombosis (ISCVT). Stroke 2004; 35: 664-70

8. Van den Bergh WM, van der Schaaf I, van Gijn J. The spectrum of presentations of venous infarction caused by deep cerebral vein thrombosis. Neurology 2005; 65: 192-96.

9. Adam SS, Key NS, Greenberg CS (March 2009). "D-dimer antigen: current concepts 
and future prospects". Blood 113 (13): 2878-87 Wells PS, Anderson DR, Rodger M, et al. (2003). "Evaluation of D-dimer in the diagnosis of suspected deep-vein thrombosis". N. Engl. J. Med. 349 (13): 1227-35

10. Wells PS, Anderson DR, Rodger M, et al. (2003). "Evaluation of D-dimer in the diagnosis of suspected deep-vein thrombosis". N. Engl. J. Med. 349 (13): 1227-35

11. Rathbun, SW; TL Whitsett, SK Vesely, GE Raskob (2004). "Clinical utility of Ddimer in patients with suspected pulmonary embolism and nondiagnostic lung scans or negative CT findings". Chest 125 (3):

$851-$

855. doi:10.1378/chest.125.3.851.

PMC 1215466. PMID 15006941.

12. Schrecengost JE, LeGallo RD, Boyd JC et al. (September 2003). "Comparison of diagnostic accuracies in outpatients and hospitalized patients of D-dimer testing for the evaluation of suspected pulmonary embolism". Clinical Chemistry 49 (9): 1483-1490. doi:10.1373/49.9.1483.

PMID 12928229

13. $17^{\text {th }}$ edition Harrison's Principles Of Internal Medicine. Chapter 110, pg 729

14. Consultative hemostasis and thrombosis By Craig S. Kitchens, Barbara M. Alving, Craig M. Kessle

15. Gates PC. Cerebral venous thrombosis: CMAJ 142 (3) 122-125; 1991. 